CARDIOVASCULAR MEDICINE

\title{
Lack of a close confidant, but not depression, predicts further cardiac events after myocardial infarction
}

\author{
C M Dickens, L McGowan, C Percival, J Douglas, B Tomenson, L Cotter, A Heagerty, F H Creed
}

Heart 2004;90:518-522. doi: 10.1136/hrt.2003.011668

See end of article for authors' affiliations .....................

Correspondence to: Dr C Dickens, Department of Psychiatry, Rawnsley Building, Manchester Royal Infirmary, Oxford Road, Manchester M13 9WL, UK; c.dickens@ man.ac.uk

Accepted 2 September 2003

\begin{abstract}
Objective: To assess the role of depression and lack of social support before myocardial infarction (MI) in determining outcome in a large representative sample of patients admitted after $\mathrm{MI}$ in the UK. Design: Prospective cohort design.

Patients: 1034 consecutive patients were screened 3-4 days after MI.

Main outcome measures: Mortality and further cardiac events over one year after an MI.

Results: At 12 months' follow up mortality and further cardiac events were assessed in 583 of 654 eligible patients (90\% response); 140 of 589 for whom baseline data were collected $(23.8 \%$ ) were depressed before their MI. Patients who were depressed before their MI were not more likely to die (mortality $5.2 \% v$ $5.0 \%$ of non-depressed patients) or suffer further cardiac events (cardiac events rate $20.7 \%$ v 20.3\% of non-depressed patients). After controlling for demographic factors and severity of $\mathrm{Ml}$, the absence of a close confidant predicted further cardiac events (hazard ratio $0.57, p=0.022$ ).

Conclusion: Lack of a close confidant but not depression before MI was associated with adverse outcome after Ml in this sample. This association may be mediated by unhealthy behaviours and lack of compliance with medical recommendations, but it is also compatible with difficulties in early life leading to heart disease.
\end{abstract}

D epression after myocardial infarction (MI) predicts subsequent mortality and morbidity, ${ }^{1}$ but the depression may have been present before the MI in a substantial proportion of patients. ${ }^{23}$ Pre-existing depression is known to be associated with an increased incidence of $\mathrm{MI}^{4}$ and with risk factors for poor outcome after MI (lack of social support, female sex, ${ }^{5}$ and continued smoking). ${ }^{6-9}$ Thus, the negative influence of depression on outcome after MI may be attributable to pre-existing depression.

One previous study reported that depression in the six months before MI did not predict worse outcome, despite being associated with more severe MIs (higher Killip class) and with post-MI depression. ${ }^{2}$ It was depression measured approximately one week after MI that predicted an increased mortality. Understanding the importance of psychosocial risk factors present before MI may contribute to our understanding of the mechanisms by which depression exerts its subsequent adverse influence on outcome.

We have performed a large prospective study to investigate the impact of psychosocial risk factors that precede the MI on fatal and non-fatal physical outcomes over the subsequent 12 months. We tested the hypothesis that depression, lack of social support, or both before MI would be associated with a more adverse outcome (cardiac deaths and non-fatal cardiac events) over the 12 months after MI.

\section{METHODS \\ Sample}

Consecutive patients with suspected MI admitted to four inner city hospitals between October 1997 and November 1999 were screened. Patients were included if they met World Health Organization criteria for MI during their hospital admission. ${ }^{10}$ Two of the following were required: history of typical chest pain; characteristic ECG changes; or a serial increase in creatine kinase to greater than twice normal limits. Patients were excluded if they: were too unwell to complete the assessment even with assistance from the researcher; suffered from a serious comorbid medical condi- tion; were insufficiently fluent in English; were over 80 years of age; or lived outside the catchment areas of the hospitals (located in Manchester and Salford) used for recruitment of subjects.

\section{Baseline assessments}

Suitable patients were assessed on average (SD) 3.6 (2.1) days after the MI. We recorded: (1) the demographic factors age, sex, marital status, and years of education; (2) smoking and use of alcohol and illegal drugs; (3) medical history of previous or family history of cardiovascular disease, and psychiatric history; and (4) details of separation from parents during childhood. Socioeconomic status was recorded according to the criteria of Goldthorpe and Hope, ${ }^{11}$ based on current or past employment of the patient or partner. Severity of angina before MI was assessed with the Rose angina questionnaire. ${ }^{12}$

Severity of the index MI was assessed with three measures:

- Killip class, ${ }^{13}$ a brief scale rating left ventricular function based on the presence of pulmonary rales, third heart sound gallop, and peripheral hypoperfusion. High scores indicate worse left ventricular function. This information was taken from the hospital records, which were based on the findings of physical examinations by a cardiologist.

- Creatine kinase concentrations, recorded over the three days after MI.

- Medication on discharge from hospital.

Blood pressure on admission and any serious cardiac complications (further MI, extension of MI, cardiac arrest) during the index admission or coronary artery bypass graft (CABG) performed during index admission were recorded from the medical records.

Abbreviations: $C A B G$, coronary artery bypass graft; HADS, hospital anxiety and depression scale; MI, myocardial infarction 
Social support was assessed in terms of whether patients had a close confidant - that is, someone with whom the patient had regular contact (at least once a month) and with whom he or she could share sensitive personal information and gain support. ${ }^{13}$

Anxiety and depression were assessed primarily with the hospital anxiety and depression scale (HADS). ${ }^{14}$ This questionnaire was developed specifically for use in medically ill populations and excludes bodily symptoms such as sleep disturbance, fatigue, and pain that may be caused by physical illness. Patients were instructed to complete the HADS questionnaire to reflect their mental state in the week before the MI. To validate the HADS questionnaire in this population we interviewed a subsample of 314 patients by using the Schedules for clinical assessment in neuropsychiatry ${ }^{15}$ to assess mental disorder. ${ }^{13}$ A HADS total combined anxiety and depression score of 17 or above had the best sensitivity $(87.7 \%)$ and specificity $(84.7 \%)$ for depressive disorder, so this cut off score was used to identify cases of depression in the whole study population $(\mathrm{n}=589)$. The first 121 patients also completed the HADS to reflect their symptoms at the time of the assessment a few days after their MI.

\section{Outcome}

The main outcomes were death from cardiac cause and further cardiac events during the 12 month period after discharge from hospital. Further cardiac events were death attributable to a cardiac cause (information from death certificate or a general practitioner or hospital records), an inpatient stay or an accident and emergency department visit as the result of an acute cardiac problem, and an angioplasty or CABG after an acute deterioration in cardiological state. Hospital attendances were only attributed to a definite cardiac event if so confirmed by inspecting the records of the findings on physical examination of the attending doctors and the results of any special investigations, such as blood tests, chest radiography, and ECGs. Presentations to hospital with chest pain that was not confirmed as cardiac after investigation or that led to angioplasty or CABG as a routine (non-acute) procedure were not classified as cardiac events.

\section{Statistical analysis}

To assess the impact of psychosocial variables on risk of further cardiac events, Cox's regression analysis was performed. Baseline demographic and physical variables that were significantly or nearly significantly $(\mathrm{p}<0.2)$ associated with further cardiac events, together with any other demographic and physical variables that have been associated with cardiovascular disease in previous research (smoking, socioeconomic status, educational status, and medication on discharge), were entered as independent variables. The baseline variables were entered in four consecutive blocks. Block l comprised age, sex, ethnicity, socioeconomic status, marital status, and years of education. Block 2 comprised number of previous MIs, history of prior angina, smoking, blood pressure on admission to hospital, Killip class, creatine kinase concentrations, and cardiac complications during index admission. Block 3 comprised medication on discharge. Block 4 comprised HAD total scores, presence of a close confidant, and separation from a parent during childhood. Forward stepwise selection of variables within each block was used, with mean substitution for missing values.

This study complied with the Declaration of Helsinki. Full ethical permission was granted by local research ethical committees (ethics application numbers: NOR/98/021, CM/ 97/090, 98090).

\section{RESULTS}

\section{Baseline variables}

This study screened 1034 consecutive patients. Of those screened, 380 were excluded for the following reasons: $>80$ years of age $(n=98)$; died before assessment $(n=43)$; too frail $(\mathrm{n}=40)$; other serious physical or mental disorder $(\mathrm{n}=37)$; cognitive impairment $(\mathrm{n}=32)$; living out of area $(n=26)$; severe sensory impairment $(n=13)$; insufficient English ( $\mathrm{n}=12)$; and other, such as late diagnosis or missed $(n=79)$. Six hundred and fifty four patients fulfilled inclusion and exclusion criteria and were eligible for the study. Sixty five patients (10\% of those eligible) refused to participate, leaving 589 patients on whom baseline data were collected. There were no significant differences with regard to age, sex, and severity of MI between patients who consented to take part in the study and those who refused.

The mean (SD) age of the sample was 60 (11.1) years; 414 $(70.3 \%)$ were men. Four hundred and ninety three patients $(84 \%)$ were admitted after their first MI, 74 with their second, 17 with their third, three with their fourth, and two with their fifth MI. One hundred forty $(23.8 \%)$ had depressive disorder (HADS score $\geqslant 17$ ).

The mean (SD) HAD score for anxiety was 6.61 (4.8) and for depression was $4.27(3.80)$ as scored to reflect mood in the week before the MI. For the 120 patients who completed the HADS for the week before as well as the few days after the MI, HAD scores before and after MI were closely correlated $(r=0.75, \mathrm{df}=120, \mathrm{p}<0.0005)$, though there was a significant increase from a mean (SD) of 3.7 (3.2) before to 4.2 (4.0) after $(t=2.1$, df $=120$, $\mathrm{p}=0.04)$.

\section{Outcome measures \\ Mortality}

Five hundred and eighty three patients (99\%) were successfully traced over the 12 month follow up period; 38 (6.5\%) died during this time (29 of cardiac causes).

Patients who died of cardiac causes were older than those who survived (mean (SD) ages 67.0 (8.4) v 59.5 (11.1) years, $t=3.6, \mathrm{p}<0.0005)$, but otherwise had similar demographic characteristics. They were more likely than the remainder to have had a previous MI $(9(31 \%) \vee 86(16 \%)$, $\mathrm{p}=0.040)$ and to have smoked $(14(48 \%) \vee 171(32 \%)$, $\mathrm{p}=0.049$ ). They had more severe MIs (7 (24.1\%) with Killip class 3 compared with $18(3.3 \%), \mathrm{p}<0.0005)$ and tended to suffer serious cardiac complications during their index admissions (5 (21\%) v $37(11 \%), \mathrm{p}=0.18)$.

Seven of $85(8.2 \%)$ patients without a close confidant and 21 of $471(4.5 \%)$ with a close confidant died of cardiac causes (Fisher's exact test, $\mathrm{p}=0.17$ ). Cardiac death was not associated with depression before MI $(\mathrm{p}=1.0)$, psychiatric history $(\mathrm{p}=0.24)$, or separation from either parent during childhood $(p=1.0)$. Since there were so few deaths attributable to cardiac causes multivariate analyses were not performed.

\section{Further cardiac events}

One hundred and eighteen patients suffered a definite cardiac event during follow up. Table 1 shows the characteristics of these patients. They were not more likely to have depression before MI than the remainder but they were slightly less likely to have a close confidant (Fisher's exact test, $\mathrm{p}=0.080$ ). There were no differences in prescription of $\alpha$ blockers, cholesterol lowering statins, warfarin, antidepressants, anticonvulsants, or hypnotics.

In the Cox's regression analysis the risk (hazard ratios) of cardiac events approximately doubled with each of the following characteristics: female sex, a history of angina, 
Table 1 Comparison of baseline characteristics of patients who did and did not experience a further cardiac event $(n=574)$

\begin{tabular}{|c|c|c|c|}
\hline & No cardiac event & Cardiac event & Significance \\
\hline Number of subjects & 456 & 118 & \\
\hline Age (years) & $59.2(11.2)$ & $62.4(10.4)$ & $t=2.87, p=0.004$ \\
\hline \multicolumn{4}{|l|}{ Sex } \\
\hline Male & $336(73.7 \%)$ & $72(61.0 \%)$ & \multirow[t]{2}{*}{$P=0.009$} \\
\hline Female & $120(26.3 \%)$ & $46(39.9 \%)$ & \\
\hline \multicolumn{4}{|l|}{ Ethnicity } \\
\hline White & 433 (95.0\%) & 112 (94.9\%) & \multirow{4}{*}{$\begin{array}{l}\chi^{2}=8.8 \\
d f=3 \\
p=0.033\end{array}$} \\
\hline Asian & $18(3.9 \%)$ & $1(0.8 \%)$ & \\
\hline Afro-Caribbean & $3(0.7 \%)$ & $4(3.4 \%)$ & \\
\hline Other & $2(0.4 \%)$ & $1(0.8 \%)$ & \\
\hline \multicolumn{4}{|l|}{ Marital status } \\
\hline Single & $44(9.6 \%)$ & $11(9.3 \%)$ & \\
\hline Married/cohabiting & $302(66.2 \%)$ & $66(55.9 \%)$ & \\
\hline Widowed & $61(13.4 \%)$ & $23(19.5 \%)$ & $\begin{array}{l}\chi^{2}=5.6 \\
d f=3\end{array}$ \\
\hline Divorced/separated & $49(10.7 \%)$ & $18(15.3 \%)$ & \multirow{2}{*}{$\begin{array}{l}p=0.13 \\
p=0.080\end{array}$} \\
\hline Close confidant & $380(86.2 \%)$ & $91(79.1 \%)$ & \\
\hline Educated to GCSE or more & $123(27.0 \%)$ & $26(22.0 \%)$ & \multirow{2}{*}{$\begin{array}{l}p=0.080 \\
p=0.29\end{array}$} \\
\hline \multicolumn{3}{|l|}{ Job class* ${ }^{*}$} & \\
\hline Class 1-21 & $192(43.6 \%)$ & $48(41.7 \%)$ & \multirow[t]{2}{*}{$p=0.75$} \\
\hline Class $22-35$ & $248(56.4 \%)$ & $67(58.3 \%)$ & \\
\hline Separation from mother in childhood & $72(16.3 \%)$ & $22(19.8 \%)$ & $p=0.40$ \\
\hline Separation from father in childhood & $113(25.4 \%)$ & $25(22.5 \%)$ & \multirow{2}{*}{$p=0.62$} \\
\hline Psychiatric history & $162(35.5 \%)$ & $40(34.2 \%)$ & \\
\hline History of angina & $89(19.5 \%)$ & $46(39.0 \%)$ & $\begin{array}{l}p=0.83 \\
p<0.0005\end{array}$ \\
\hline \multicolumn{4}{|l|}{ Severity of angina (Rose) $n=128$} \\
\hline Moderate & $59(67.8 \%)$ & $26(63.4 \%)$ & $p=0.69$ \\
\hline Severe & $29(32.2 \%)$ & $14(36.6 \%)$ & \\
\hline Smoking history & & & \\
\hline Never smoked & $79(17.4 \%)$ & $23(19.7)$ & $\chi^{2}=3.68$ \\
\hline Current smoker & $234(51.7 \%)$ & $49(41.9 \%)$ & $\mathrm{df}=2$ \\
\hline Past smoker & $140(30.9 \%)$ & $45(38.5 \%)$ & $p=0.16$ \\
\hline Illegal drug use & $18(4.0 \%)$ & $5(4.3 \%)$ & $p=0.80$ \\
\hline Family history of MI & $264(59.6 \%)$ & $62(56.4 \%)$ & $p=0.59$ \\
\hline Number of previous Mls & & & \\
\hline 0 & $389(85.3 \%)$ & $90(76.3 \%)$ & Linear trend \\
\hline 1 & $56(12.3 \%)$ & $17(14.4 \%)$ & $\chi^{2}=14.8$ \\
\hline 2 & $11(2.4 \%)$ & $6(5.1 \%)$ & $\mathrm{df}=1$ \\
\hline 3 & $0(0 \%)$ & $3(2.5 \%)$ & $p<0.0005$ \\
\hline 4 & $0(0 \%)$ & $2(1.6 \%)$ & \\
\hline Previous CABG & $11(5.3 \%)$ & $9(13.8 \%)$ & $p=0.030$ \\
\hline Depressed (HADS total $\geqslant 17$ ) & $107(23.5 \%)$ & $28(23.7 \%)$ & $p=1.0$ \\
\hline Blood pressure on admission $(\mathrm{mm} \mathrm{Hg})$ & & & \\
\hline Diastolic & $69.5(12.6)$ & $71.3(13.4)$ & $t=1.39, p=0.17$ \\
\hline Systolic & $121.2(16.7)$ & $122.5(21.3)$ & $t=0.61, p=0.54$ \\
\hline Severity of index MI (Killip class) & & & \\
\hline 1 & 332 (73.0\%) & $61(52.1 \%)$ & $\chi^{2}=29.7$ \\
\hline 2 & $112(24.6 \%)$ & $42(35.9 \%)$ & $\mathrm{df}=2$ \\
\hline 3 & $11(2.4 \%)$ & $14(12.0 \%)$ & $p<0.0005$ \\
\hline Creatine kinase $†$ (iv/l) & & & \\
\hline First sample & $586.6(875.3)$ & $609.4(952.4)$ & $p=0.56$ \\
\hline Second sample & $1203.6(1184.0)$ & $1381.7(1249.2)$ & $p=0.10$ \\
\hline Third sample & $918.9(1129.5)$ & $1050.0(1312.4)$ & $p=0.08$ \\
\hline Thrombolysis & $327(71.7 \%)$ & $79(66.9 \%)$ & $p=0.31$ \\
\hline $\begin{array}{l}\text { Severe cardiac complications during index } \\
\text { admission }\end{array}$ & $24(8.9 \%)$ & $18(21.2 \%)$ & $p=0.004$ \\
\hline CABG during index admission & $11(2.4 \%)$ & $1(0.9 \%)$ & FET, $p=0.48$ \\
\hline $\begin{array}{l}\text { Discharge medication } \\
\text { Aspirin }\end{array}$ & & & \\
\hline Aspirin & $434(95.8 \%)$ & $96(88.9 \%)$ & $p=0.009$ \\
\hline$\beta$ Blockers & 285 (62.9\%) & $48(44.4 \%)$ & $p=0.001$ \\
\hline Calcium channel blockers & $81(17.9 \%)$ & $31(28.7 \%)$ & $p=0.016$ \\
\hline ACE inhibitors & $191(42.3 \%)$ & $59(54.6 \%)$ & $p=0.024$ \\
\hline Nitrates & $272(60.2 \%)$ & $77(71.3 \%)$ & $p=0.036$ \\
\hline Statins & $337(74.6 \%)$ & $73(67.6 \%)$ & $\mathrm{p}=0.15$ \\
\hline Diuretics & $72(16.0 \%)$ & $35(32.4 \%)$ & $p<0.0005$ \\
\hline $\begin{array}{l}\text { *Job classes } 1-21 \text { reflect higher socioecono } \\
\text { Whitney U test in view of degree of non-norn } \\
\text { was used. } \\
\text { ACE, angiotensin converting enzyme; CABG } \\
\text { Certificate of Secondary Education lequival } \\
\text { scale; } M I \text {, myocardial infarction. }\end{array}$ & $\begin{array}{l}\text { oronary artery byp } \\
\text { to } 12 \text { years of edu }\end{array}$ & $\begin{array}{l}\text { flect lower status; } \\
\text { ariables. Unequal } \\
\text { graft; FET, Fisher' } \\
\text { tion); HADS, hosp }\end{array}$ & $\begin{array}{l}\text { mpared by Mann- } \\
\text { ance version of the } t \text { tes } \\
\text { act test; GCSE, Genero } \\
\text { anxiety and depressior }\end{array}$ \\
\hline
\end{tabular}

and prescription calcium channel blockers (table 2). Each unitary increase in Killip class (that is, from 1 to 2 or from 2 to 3 ) increased the hazard ratio for further events during the 12 months after MI by a factor of 1.8. Patients with a close confidant had only half the risk of a further cardiac event of those without a confidant. Depression did not significantly improve the model $(\mathrm{p}=0.41)$.

Post hoc analyses indicated that patients without a close confidant were more likely to be presenting with their second or subsequent MI (26\% $v 14.1 \%$, Fisher's exact test, 
Table 2 Factors predicting time to further cardiac events during the follow up period in whole sample of patients recruited $(n=574)$

\begin{tabular}{llll}
\hline Variable & Hazard ratio & 95\% confidence interval & p Value \\
\hline Female sex & 1.83 & 1.23 to 2.71 & 0.0026 \\
History of angina (yes/no) & 1.83 & 1.21 to 2.75 & 0.0039 \\
Killip class (1, 2, or 3) & 1.80 & 1.35 to 2.41 & 0.0001 \\
Cardiac complication during index admission & 2.44 & 1.38 to 4.34 & 0.0023 \\
Calcium channel blockers (yes/no) & 1.61 & 1.06 to 2.47 & 0.027 \\
Close confidant (yes/no) & 0.57 & 0.35 to 0.92 & 0.022 \\
\hline
\end{tabular}

$\mathrm{p}=0.015)$ but they did not have more severe index MIs (Killip class). Patients without a close confidant were more likely to have been separated from both parents in childhood than patients with a close confidant $(26 \%$ v $10 \%$, $\mathrm{p}=0.0003)$, more likely to drink alcohol four or more times a week $(28 \% \vee 15 \%$, Fisher's exact test, $p=0.012)$, more likely to consume more than six drinks in a single session at least once a week $(22 \% v 14 \%, \mathrm{p}=0.049)$, and more likely to use illegal drugs $(8 \% \vee 3 \%, \mathrm{p}=0.034)$. There were non-significant trends for lack of a close confidant to be associated with current smoking $(60 \% v$ $48 \%$, respectively, $\mathrm{p}=0.058$ ). Lack of a close confidant was not associated with depression (Fisher's exact test, $p=0.27)$. None of these variables that were associated with lack of a close confidant were themselves significantly associated with increased cardiac events during the follow up year.

\section{DISCUSSION}

This study is the largest prospective cohort study of the impact of psychosocial variables preceding an MI on subsequent mortality and further cardiac events in a representative sample. There are two main findings. We failed to find an association between depression before MI and subsequent mortality or cardiac events, in spite of the high prevalence of depression. We did find that having a close confidant approximately halved the risk of having a subsequent cardiac event, even after controlling for demographic and coronary risk factors, severity of MI, and discharge medication.

Our study differed from most other work in the area because we assessed depression during the week before the MI rather than the week(s) after. We did this because we suspected that chronic depression before MI, associated with social difficulties, would be most closely associated with poor outcome $^{316}$ We were wrong; in this study depression that preceded MI was not associated with subsequent increased mortality or cardiac events. This finding is consistent with the findings of Lesperance and colleagues, ${ }^{2}$ who found that it was only depression after the MI that was associated with increased mortality. Also, other studies showing that depression increases mortality have all measured depression in the days or weeks after MI. ${ }^{1}$ Thus, despite there being a close overall correlation between HAD scores before and after MI, the greatest risk for poor outcome appears to lie with depression detected after MI. One possible explanation is that the risk is greatest in patients whose depression starts in the days after MI when an increase in sympathetic tone interacts with a vulnerable myocardium. ${ }^{17}$ Our sample of patients who developed new episodes of depression in the week after MI was too small to assess the effect of this change.

Most previous work has been concerned only with mortality. This was so low in our sample that we could not perform a multivariate analysis to identify the predictors of mortality. This presumably reflects improved treatment. A longer follow up is planned. Other possible reasons for not confirming the Canadian results ${ }^{1}$ are a different measure of depression and a different sample-our sample included more women than some previous studies. One recent study found that the relation between post-MI depression and increased mortality held only in the group older than 65 years,$^{18}$ so small differences in sampling may lead to different results.

Our finding that lack of a close confidant was associated with further cardiac events accords with several previous studies. Our measure concerned the degree of intimacy of close relationships rather than the number of social contacts; the close relationships may be protective of health ${ }^{19}$ and may promote recovery from depression. ${ }^{20}$ The mechanism by which having a close confidant had an effect on cardiac outcome in our patients remains obscure. We found that patients who did not have a close confidant were more likely to smoke and drink heavily, though these behavioural variables did not predict poor outcome in multivariate analysis and are unlikely to account for the negative effects of not having a close confidant. It is possible that separation from parents in childhood confounded the association of lack of close confidant with worse physical outcome after MI. Separation from parents in childhood may reduce the chances of forming an intimate relationship in adulthood and may increase the risk for ischaemic heart disease-for example, by its association with low birth weight. Alternatively, those who do not have a close confidant may delay seeking treatment for $\mathrm{MI}^{21}$ or may be less likely to adhere to treatment after MI. ${ }^{22}$

Our study has a number of methodological strengths. We recruited a large, representative sample of patients admitted after MI and assessed them with robust measures. In addition, we took great care in obtaining accurate follow up data on a very high proportion of patients. By using multivariate statistical techniques we were able to control for the influence of conventional risk factors (for example, smoking and medication for diabetes and hypertension) on outcome. We deliberately used a measure of depression that prevents bodily symptoms confounding the association between depression and poor outcome. ${ }^{23}$ Though we validated our questionnaire assessment of depression against an ideal standardised research interview, it must be recognised that our assessment of depression was retrospective and as such the exact relation in time of the depression and the MI may be subject to recall bias. Care should be exercised in the interpretation of our findings. In retrospect, had we measured depression one week after MI, we would have been able to compare our results with those of others. It is also important to remember that we studied only patients who survived their MI until the time of our assessment. Many would have died before reaching hospital and this would have been a major determinant of our sample. Future studies should consider measuring delay in treatment 
seeking as a possible mediating factor between social support and outcome.

Despite the negative results with regard to the impact of depression on cardiac outcome, it should be stressed that this does not mean that physicians and cardiologists can ignore depression. The high prevalence of depression in our sample testifies to the importance of depression as a risk factor for developing an MI. ${ }^{24}$ Primary prevention of coronary artery disease needs to encompass this risk factor. With regard to secondary prevention, depressed people, as well as those without a close confidant, are less likely to give up smoking after an MI. ${ }^{8}$ Improving social support and depression therefore carry considerable potential to improve primary and secondary prevention of MI.

\section{ACKNOWLEDGEMENTS}

This study was funded by the British Heart Foundation and the Medical Research Council of the UK. We thank Dick Heller, Professor of Public Health, Manchester University, for his help with the preparation of this paper.

\section{Authors' affiliations}

C M Dickens, L McGowan, C Percival, J Douglas, B Tomenson, F H Creed, Psychological Medicine Research Group, Department of Psychiatry, Manchester University, Manchester, UK

L Cotter, A Heagerty, Department of Cardiology, Manchester Royal Infirmary, Manchester, UK

\section{REFERENCES}

1 Frasure-Smith N, Lesperance F, Talajic M. Depression following myocardial infarction: impact on 6-month survival. JAMA 1993;270:1819-25.

2 Lesperance F, Frasure-Smith N, Talajic M. Major depression before and after myocardial infarction: its nature and consequences. Psychosom Med 1996:58:99-110.

3 Lloyd GG, Cawley RH. Psychiatric morbidity after myocardial infarction. QJM 1982;201:33-42.

4 Barefoot JC, Schroll M. Symptoms of depression, acute myocardial infarction and total mortality in a community sample. Circulation 1996;93:1976-80.

5 Meltzer H, Gill B, Petticrew M, et al. The prevalence of psychiatric morbidity among adults living in private households. 1. OPCS surveys of psychiatric morbidity in Great Britain. London: HMSO, 1995.
6 Brown G, Harris T. Social origins of depression: a study of psychiatric disorder in women. London: Tavistock, 1978.

7 Blazer DG, Kessler RC, McGonagle KA, et al. The prevalence and distribution of major depression in a national community sample: the national comorbidity study. Am J Psychiatry 1994;151:979-86.

8 Lloyd GC, Cawley RH. Smoking habits after myocardial infarction. J R Coll Physicians Lond 1980;14:224-6.

9 Berkman LF, Leo-Summers L, Horwitz RI. Emotional support after myocardial infarction: a prospective, population-based study of the elderly. Ann Intern Med 1992;117:1003-9.

10 Tunstall-Pedoe H, Kuulasmaa K, Amouyel P, et al. Myocardial infarction and coronary deaths in the World Health Organization Monica project: registration procedures, event rates and case-fatality rates in 38 populations from 21 countries in four continents. Circulation 1994;90:583-612.

11 Goldthorpe JH, Hope K. The social grading of occupations: a new approach and scale. London: Oxford University Press, 1974.

12 Rose GA. The diagnosis of ischaemic heart pain and intermittent claudication in field surveys. Bull WHO 1962;27:645-58.

13 Killip T, Kimball JT. Treatment of myocardial infarction in a coronary care unit: a two year experience with 250 patients. Am J Cardiol 1967;20:457-64.

14 Zigmond AS, Snaith RP. The hospital anxiety and depression scale. Acta Psychiatr Scand 1983;67:361-70.

15 Anon. World Health Organization Division of Mental Health. Schedule for clinical assessment in neuropsychiatry, version 2. Arlington: American Psychiatric Press, 1996.

16 Lloyd GG, Cawley RH. Distress or illness? A study of the psychological symptoms after myocardial infarction. Br J Psychiatry 1983;142:120-5.

17 Musselman DL, Evans DL, Nemeroff CB. The relationship of depression to cardiovascular disease. Arch Gen Psychiatry 1998;55:580-92.

18 Shiotani I, Sato H, Kinjo K, et al. Depressive symptoms predict 12 month prognosis in elderly patients with acute myocardial infarction. J Cardiovasc Risk 2002;9:153-60.

19 Mendes de Leon CF. Depression and social support in recovery from myocardial infarction: confounding and confusing. Psychosom Med 1999:61:738-9.

20 Lesperance $\mathbf{F}$, Frasure-Smith N, Talajic $M$, et al. Five-year risk of cardiac mortality in relation to initial severity and one-year changes in depression symptoms after myocardial infarction. Circulation 2002;105:1049-53.

21 Schwarz B, Schoberberger R, Rieder A, et al. Factors delaying treatment of acute myocardial infarction. Eur Heart J 1994;15:1595-8.

22 DiMatteo MR, Lepper HS, Croghan TW. Depression is a risk factor for noncompliance with medical treatment. Arch Intern Med 2000;160:2101-7.

23 Irvine J, Baker B, Smith J, et al. Poor adherence to placebo or amiodarone therapy predicts mortality: results from the CAMIAT study. Canadian amiodarone myocardial infarction arrhythmia trial. Psychosom Med 1999:61:566-75

24 Hemingway $H$, Marmot $M$. Evidence based cardiology: psychosocial factors in the aetiology and prognosis of coronary heart disease. Systematic review of prospective cohort studies. BMJ 1999;318:1460-7.

\section{ELECTRONIC PAGES}

\section{Heart Online case reports: www.heartinl.com}

7

he follow electronic only articles are published in conjunction with this issue of Heart.

\section{Cardiac actinomycosis in a patient presenting with acute cardiac tamponade and a mass mimicking pericardial tumour}

L Jánoskuti, M Lengyel, T Fenyvesi

A case of pericardial actinomycosis mimicking a pericardial tumour is reported. After the appearance of non-specific subpleural pulmonary nodules, a 48 year old woman presented with fever and clinical signs of pericardial tamponade. Subxiphoid pericardiotomy yielded a culture negative fluid and inflammatory reactive histopathology in the pericardial biopsy specimen. Because of suspected infection cefamandole was administered for 10 days and the patient became afebrile. The pericardial effusion recurred with no clinical signs two weeks later. Steroid medication resulted in rapid regression of the pericardial effusion. Subsequent echocardiography controls showed a tumour-like pericardial mass, confirmed by cardiac magnetic imaging. Surgical exploration led to the final histological diagnosis of actinomycosis. After high dose and long term penicillin $G$ treatment the patient recovered fully with no recurrence during two years' follow up.

(Heart 2004;90:e27) www.heartjnl.com/cgi/content/full/90/ $5 / \mathrm{e} 27$

\section{Knotting of a pulmonary artery catheter in the superior vena cava: surgical removal and a word of caution}

G P Georghiou, B A Vidne, E Raanani

A case of postoperative pulmonary artery catheterisation complicated by knotting of the catheter (Swan-Ganz) within the superior vena cava is described. The catheter was cut off at the skin entry site. The remainder, together with the knot, was pulled out through a purse string incision in the superior vena cava.

(Heart 2004;90:e28) www.heartjnl.com/cgi/content/full/90/ $5 / \mathrm{e} 28$ 